\title{
Application of simulated lidar scanning patterns to constrained Gaussian turbulence fields for load validation
}

\section{Dimitrov, Nikolay Krasimirov; Natarajan, Anand}

Published in:
Wind Energy

Link to article, DOI:

10.1002/we.1992

Publication date:

2017

Document Version

Publisher's PDF, also known as Version of record

Link back to DTU Orbit

Citation (APA):

Dimitrov, N. K., \& Natarajan, A. (2017). Application of simulated lidar scanning patterns to constrained Gaussian turbulence fields for load validation. Wind Energy, 20(1), 79-95. https://doi.org/10.1002/we.1992

\section{General rights}

Copyright and moral rights for the publications made accessible in the public portal are retained by the authors and/or other copyright owners and it is a condition of accessing publications that users recognise and abide by the legal requirements associated with these rights.

- Users may download and print one copy of any publication from the public portal for the purpose of private study or research.

- You may not further distribute the material or use it for any profit-making activity or commercial gain

- You may freely distribute the URL identifying the publication in the public portal 


\title{
Application of simulated lidar scanning patterns to constrained Gaussian turbulence fields for load validation
}

\author{
Nikolay Dimitrov and Anand Natarajan \\ Department of Wind Energy, Technical University of Denmark, Risoe campus, Roskilde, Denmark
}

\begin{abstract}
We demonstrate a method for incorporating wind velocity measurements from multiple-point scanning lidars into threedimensional wind turbulence time series serving as input to wind turbine load simulations. Simulated lidar scanning patterns are implemented by imposing constraints on randomly generated Gaussian turbulence fields in compliance with the Mann model for neutral stability. The expected efficiency of various scanning patterns is estimated by means of the explained variance associated with the constrained field. A numerical study is made using the HAWC2 aeroelastic software, whereby the constrained turbulence wind time series serves as input to load simulations on a $10 \mathrm{MW}$ wind turbine model using scanning patterns simulating different lidar technologies_-pulsed lidar with one or multiple beams-and continuouswave lidars scanning in three different revolving patterns. Based on the results of this study, we assess the influence of the proposed method on the statistical uncertainty in wind turbine extreme and fatigue loads. The main conclusion is that introducing lidar measurements as turbulence constraints in load simulations may bring significant reduction in load and energy production uncertainty, not accounting for any additional uncertainty from real measurements. The constrained turbulence method is most efficient for prediction of energy production and loads governed by the turbulence intensity and the thrust force, while for other load components such as tower base side-to-side moment, the achieved reduction in uncertainty is minimal. Copyright (C) 2016 John Wiley \& Sons, Ltd.
\end{abstract}

\section{KEYWORDS}

turbulence; uncertainty; constrained; lidar; load simulations; wind measurements; load verification; random field

\section{Correspondence}

Nikolay Dimitrov, Department of Wind Energy, Technical University of Denmark, Risoe campus, Roskilde, Denmark. E-mail: nkdi@dtu.dk

\section{INTRODUCTION}

Mechanical loads on wind turbines are to a large extent driven by the variations in the stochastic wind turbulence field interacting with the turbine rotor. Because of the large volumes and length scales involved and the complexity of the turbulent flow, it is currently not possible to characterize the inflow in its entirety solely by measurements. Therefore, in numerical load simulations, the turbulent wind is usually modelled as a random three-dimensional Gaussian field with spectral properties defined by a turbulence model as e.g. the Kaimal spectrum used with the Veers method, ${ }^{1}$ or the Mann turbulence model. ${ }^{2,3}$ For the purpose of load verification, the statistical properties (mean and variance) of the generated numerical wind field are defined to match the statistical properties of time series obtained by measurements at one or few points in front of the turbine rotor. Because of the stochastic nature of the problem, the instantaneous values of a generated wind field realization will not match the true wind velocities experienced by the turbine. As a result, the outcome of a well-performed numerical load analysis will match the measured load values on a statistical basis, but there still can be significant realization-to-realization variation as a result of the uncertainty in the inflow. This uncertainty implies that a significantly large number of observations and simulations are required for a successful load verification of wind turbine prototypes.

Recent developments in remote sensing technology have made it possible to measure wind velocities at multiple points in a predefined pattern by means of multiple-beam lidars and scanning lidars. Although not being able to fully characterize 
turbulence, the multi-point measurement technology can provide significantly more information about the incoming wind velocities as compared with measurements at a single point as provided by e.g. cup or sonic anemometers. However, it needs to be shown that incorporating multi-point lidar measurements in load simulations will reduce the sample-to-sample uncertainty of numerical load simulations with respect to observed wind turbine loads. Earlier studies considered some aspects of the problem, e.g. Bierbooms and Cheng ${ }^{4}$ describe a stochastic gust model for load simulations which can be used to generate constrained time series, and Nielsen et al. ${ }^{5}$ show a method for creating constrained turbulence fields to be used in load simulations. These studies typically consider the constraints as a discrete event as e.g. coherent gust, leading to only few constraints applied within a short time period. Bierbooms and Veldkamp ${ }^{6}$ and Kim et al. ${ }^{7}$ compare measured and simulated loads using wind fields constrained at either one or two locations based on cup anemometer wind measurements. In the present paper, we explore possible benefits to load prediction by a numerical study which assesses the effect of including wind velocity time series measured by multi-point lidar in the turbulence field used for aeroelastic load simulations. Constrained Gaussian turbulence fields are generated using simulated Lidar measurements, where each instantaneous measured value is treated as a constraint. With the proposed approach, constraints can be applied over the entire volume of a turbulence field used in an aeroelastic simulation, meaning that a significant part of the turbulence in the simulations will be determined from measured values. This makes the method potentially useful for model validation tasks such as load and power curve verification, as well as many other purposes where a reduced realization-to-realization uncertainty has an importance. In the following sections, we describe the process of generating a constrained turbulence field and assess the significance of using a constrained field on the load uncertainty.

\section{GENERATING CONSTRAINED TURBULENCE FIELDS}

\subsection{Constrained Gaussian fields}

The algorithm for applying constraints on a Gaussian field uses a source, unconstrained realization of the random field, which is modified to satisfy all constraints by adding a so-called residual field, conditionally dependent on the constraints. The residual field is defined by using its most likely values to interpolate between known points (e.g. constraints), a technique which is often referred to as simple kriging, ${ }^{8}$ linear regression ${ }^{9}$ or optimal linear estimation method. ${ }^{10}$ In the succeeding texts, we give a short overview of the method, which is described in more details in Hoffman and Ribak ${ }^{11}$ and Nielsen et al. ${ }^{5}$

Consider a zero-mean, homogeneous and isotropic Gaussian random field $g(\mathbf{r}), \mathbf{r}=\{x, y, z\}$, which is defined by its power spectrum $S_{\mathrm{r}}(\mathbf{k})$ and is subjected to a set of $M$ constraints denoted by $\mathbf{H}=\left\{\left.h_{\mathrm{i}}(\mathbf{r})\right|_{r_{\mathrm{i}}}=c_{\mathrm{i}}, i=1, \ldots, M\right\}$. A constraint at a point $\mathbf{r}_{\mathrm{i}}$ is a function of $g\left(\mathbf{r}_{\mathrm{i}}\right)$, and in the present problem, this is simply the required value of the field at $\mathbf{r}_{\mathrm{i}}$, i.e. $h_{\mathrm{i}}\left(\mathbf{r}_{\mathrm{i}}\right) \equiv$ $g\left(\mathbf{r}_{\mathbf{i}}\right)=c_{\mathrm{i}}$. In order to generate a random field realization $g(\mathbf{r})$ which has a spectrum given by $S_{\mathrm{r}}(\mathbf{k})$ and satisfies the conditions in $\mathbf{H}$, two main steps are necessary: (i) generate a random, unconstrained realization $\tilde{g}(\mathbf{r})$ and (ii) impose the constraints in $\mathbf{H}$ on $\tilde{g}(\mathbf{r})$ to obtain $g(\mathbf{r})$. The residual field representing the difference between the unconstrained and the constrained fields, $\varepsilon_{\mathrm{g}}(\mathbf{r})=g(\mathbf{r})-\tilde{g}(\mathbf{r})$, is also a random Gaussian field. At all constraint locations $\mathbf{r}_{\mathrm{i}}, i=1 \ldots M$, the values of $\varepsilon_{\mathrm{g}}$ are known: $\varepsilon_{\mathrm{g}}\left(\mathbf{r}_{\mathrm{i}}\right)=c_{\mathrm{i}}-\tilde{g}\left(\mathbf{r}_{\mathrm{i}}\right)$. For all other locations, the values of $\varepsilon_{\mathrm{g}}(\mathbf{r})$ are uncertain; their probability distribution is conditional on the constraints $\mathbf{H}$ and is given by

$$
P\left[\varepsilon_{\mathrm{g}}(\mathbf{r}) \mid \mathbf{H}\right]=\frac{P\left[\varepsilon_{\mathrm{g}}(\mathbf{r}), \mathbf{H}\right]}{P[\mathbf{H}]}
$$

where $P[$.] is the multivariate normal (Gaussian) distribution. For the purpose of obtaining a realization of $g(\mathbf{r})$, a suitable choice for the uncertain points in $\varepsilon_{\mathrm{g}}(\mathbf{r})$ is their most likely values which are given by the ensemble mean of $\varepsilon_{\mathrm{g}}(\mathbf{r}) \mid \mathbf{H}$ :

$$
\bar{\varepsilon}_{\mathrm{g}}(\mathbf{r})=\left\langle\varepsilon_{\mathrm{g}}(\mathbf{r}) \mid \mathbf{H}\right\rangle=\zeta(\mathbf{r}) \mathbf{Z}^{-1}\left(\mathbf{H}-\tilde{g}_{\mathrm{c}}(\mathbf{r})\right)
$$

where $\langle$.$\rangle denotes ensemble averaging, \zeta(\mathbf{r})=\left[\left\langle g(\mathbf{r}) g\left(\mathbf{r}_{1}\right)\right\rangle,\left\langle g(\mathbf{r}) g\left(\mathbf{r}_{2}\right)\right\rangle, \ldots,\left\langle g(\mathbf{r}) g\left(\mathbf{r}_{\mathrm{M}}\right)\right\rangle\right]$ is a vector of the cross-correlations between the field and the constraints, and $\mathbf{Z}$ is the correlation matrix of the constraints set, $Z_{\mathrm{ij}}=\left\langle g\left(\mathbf{r}_{\mathrm{i}}\right) g\left(\mathbf{r}_{\mathrm{j}}\right)\right\rangle, i=1 \ldots$ $M, j=1 \ldots M$. Here, $\mathbf{H}=\left[c_{1}, c_{2}, \ldots, c_{\mathrm{M}}\right]^{T}$ is the vector with the specific constraint values, and $\tilde{g}_{\mathrm{c}}(\mathbf{r})=\tilde{g}\left(\mathbf{r}=\mathbf{r}\left(c_{\mathrm{i}}\right)\right), \quad i=$ $1 \ldots M$ are the values of the unconstrained realization corresponding to the constraints (i.e. at the same location and contemporaneous with the constraints). The variance of the residual field is independent of the constraint values and is given by

$$
\left\langle\varepsilon_{\mathrm{g}}^{2}(\mathbf{r}) \mid \mathbf{H}\right\rangle=\sigma^{2}-\zeta(\mathbf{r}) \mathbf{Z}^{-1} \zeta(\mathbf{r})^{T}
$$


where $\sigma^{2}$ is the variance of the unconstrained field. Any constrained realization can be written as a sum of the unconstrained field $\tilde{g}(\mathbf{r})$ and the mean of the residual field $\bar{\varepsilon}_{\mathrm{g}}(\mathbf{r})$ :

$$
g(\mathbf{r})=\tilde{g}(\mathbf{r})+\zeta(\mathbf{r}) \mathbf{Z}^{-1}\left[\mathbf{H}-\tilde{g}_{\mathrm{c}}(\mathbf{r})\right]
$$

Figure 1 shows an example with a one-dimensional, zero-mean and unit-variance Gaussian field $g(t)$ on which a single constraint $g(t)=3$ is imposed at $t=600 \mathrm{~s}$. As a result of the imposed constraint, at the constraint location, the mean of the field equals the constraint value, and the field variance is reduced to zero.

\subsection{Turbulence boxes with constraints modelling lidar measurements}

The wind inflow input to aeroelastic wind turbine load simulations is defined as a random, three-dimensional turbulence field discretized on a rectangular grid referred to as a turbulence box (Figure 2). The longitudinal wind direction is coincident with the longest dimension of the turbulence box (here given as the $x$ coordinate), while the $y$ and $z$ coordinates cover the dimensions of the rotor plane. As the load simulations span over a fixed time period, the mapping between the time and physical coordinates (metres) on the $x$-axis will depend on the specific average wind speed: The time step size is fixed, while the step size in metres is proportional to the mean wind speed, e.g. for a wind field with a 10 min duration and with a mean wind speed of $10 \mathrm{~m} / \mathrm{s}$, the length of the generated turbulence box will correspond to a physical dimension of $6000 \mathrm{~m}$. Along the axes parallel to the rotor plane, the size of the turbulence box normally is slightly larger than the rotor diameter, e.g. $180 \mathrm{~m}$ for a wind turbine with $178 \mathrm{~m}$ rotor diameter. The properties of the turbulence box are based on a spectral turbulence model, e.g. the Mann model, and the values are generated using Fourier simulation. In the present study, we use turbulence boxes with a grid size of $8192 \times 32 \times 32(x, y, z)$, which is adequate for a 10 min wind simulation over a large rotor.

At each grid location, the instantaneous wind velocity is regarded as a three-component vector $[u, v, w]$, which quantifies the wind velocity projection on the $x, y$ and $z$ axes respectively. The ratio of the standard deviation in the $v$ and $w$ components to the $u$ component is usually fixed by the Mann model parameters and is commonly assumed to be 0.8 and 0.5 respectively.
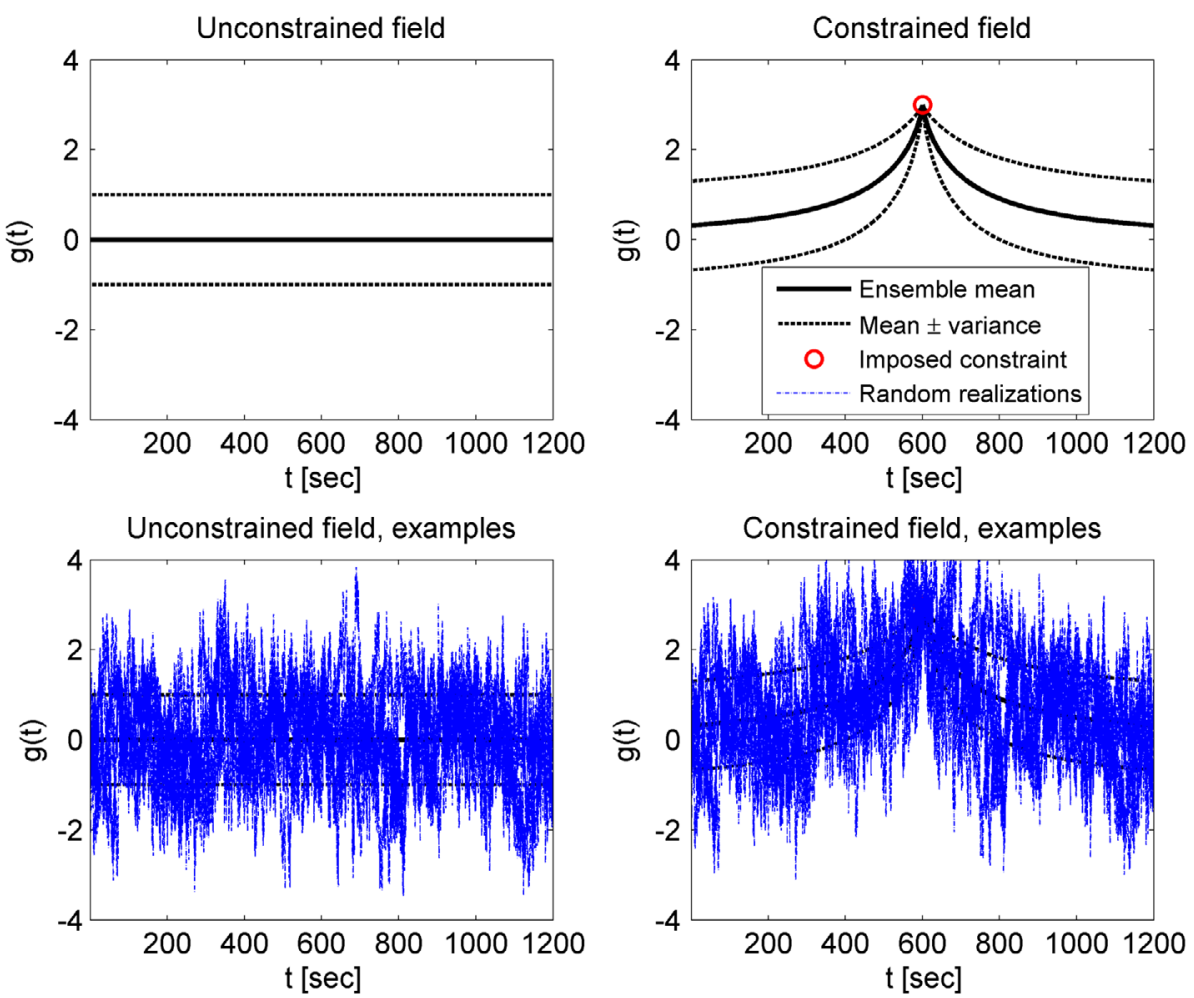

Figure 1. One-dimensional, zero-mean and unit-variance Gaussian field $g(t)$ on which a single constraint $g(t)=3$ is imposed at $t=600 \mathrm{~s}$. 


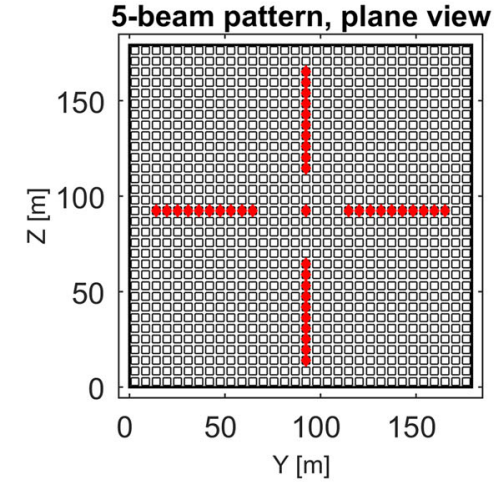

5-beam pattern, 3-D view

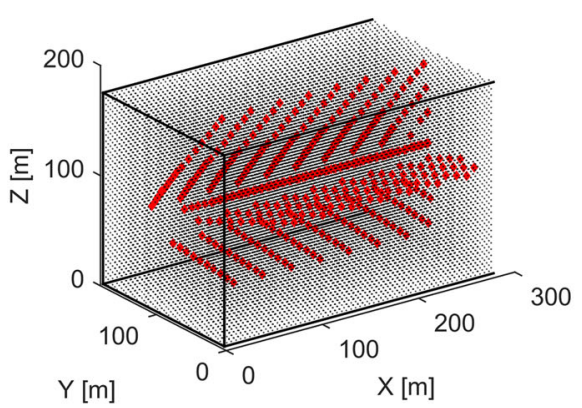

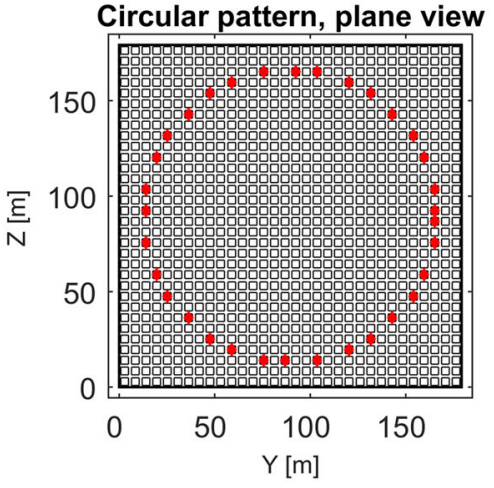

Circular pattern, 3D view

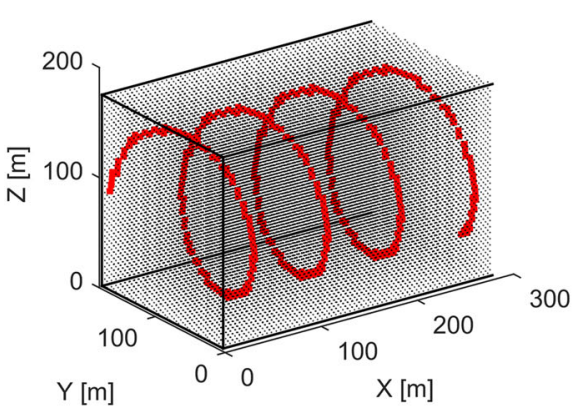

Figure 2. Constraint patterns simulating lidar measurements: five-beam pulsed lidar pattern (top left: plane view, bottom left: 3-D view); continuous-wave lidar scanning in a circular pattern (top right: plane view; bottom right: 3-D view).

The process of obtaining an accurate numerical representation of measured quantities such as wind velocities is associated with various uncertainties. Some examples of potential sources of uncertainty in the measurements from a nacellemounted lidar are

- Lidars measure the wind speed along the line-of-sight, i.e. the direction in which the laser beam is pointing. This direction is not necessarily coincident with the wind velocity coordinate system axes $u, v$ or $w$. Under certain assumptions (e.g. homogeneous flow), these line-of-sight measurements can be projected on a three-axis coordinate system. In the present paper, we consider mostly cases where we only apply constraints on the $u$ component. This choice is made in order to reduce the computation efforts. Including constraints on the $v$ and $w$ components is technically feasible but requires several times longer computations. One of the study cases included in the present work assesses the effect of constraining all turbulence components.

- The lidar measures a volume which spans several metres along the line of sight. As a result, the lidar measurements represent spatially averaged samples of the wind speed, ${ }^{12}$ which in principle can be taken into account by applying constraints on multiple points per lidar measurement. The size of the measurement volume and the maximum possible measurement frequency depends on the lidar technology: ${ }^{13}$ Continuous-wave lidars normally can measure with very high frequency, but the measurement volume increases with the focusing distance. For example, the probe length for the ZephIR 300 commercial continuous-wave lidar is $0.07 \mathrm{~m}$ at a $10 \mathrm{~m}$ measurement distance, and $7 \mathrm{~m}$ at a $100 \mathrm{~m}$ measurement distance. ${ }^{14}$ Pulsed lidars require more time between measurements, but several distance ranges can be measured simultaneously, and the measurement volume does not change with distance.

- The evolution of turbulence from the location where it is measured to the rotor plane needs to be considered. A simple approach is to use the 'frozen turbulence' hypothesis, assuming that the turbulence is advected towards the rotor with the mean wind speed.

- Turbine motion causes a shift in the measured wind speed and the measurement location. In actual lidar measurements, the turbine motion needs to be measured and accounted for.

- Lidar measurement point locations do not fit exactly on a rectangular measurement grid. The grid point where the measurement is applied can be determined by e.g. nearest-neighbour interpolation.

- Applying constraints requires the use of a turbulence spectrum, from which to determine the cross-correlations. The spectrum has either to be chosen arbitrarily, or some spectral parameter values can be fitted to measurements, with a corresponding uncertainty associated with both choices. 
Considering the challenges listed in the preceding texts, we have decided to limit the scope of the present study so that a greater emphasis can be given to the mathematical aspects of the method of creating constrained turbulence boxes. Therefore, we consider an idealized case by assuming that the lidar measurement has been used to calculate a 'known' wind speed in the longitudinal direction, at a point located on the rotor plane. Controlling the input in such a way means that we can correctly assess the effectiveness of using constraints in reducing the statistical uncertainty in the randomly generated turbulence fields, because all other sources of uncertainty are removed.

In the succeeding texts, we show as an example two constraint patterns which mimic the measurement pattern of two existing multiple-point scanning lidars:

- A multiple-beam pulsed lidar with five beams, with a centre beam pointing forward and four beams at $15^{\circ}$ angles from the centre beam. The five beams measure in sequential order. Each beam takes measurements at 10 ranges along the line of sight with a frequency of $0.2 \mathrm{~Hz}$, which results in an overall measurement frequency for all beams of $1 \mathrm{~Hz}$. The pattern is shown in Figure 2, top and bottom left. This pattern resembles the setup of the Avent 5-beam Demonstrator lidar. ${ }^{15}$

- A continuous-wave lidar with a single, rotating beam with a $15^{\circ}$ cone angle, rotating with an angular velocity of $2.3 \mathrm{rad} / \mathrm{s}$ and a measurement frequency of $11.72 \mathrm{~Hz}$. One revolution lasts $2.73 \mathrm{~s}$ and consists of 32 measurement points. The sampling frequency is chosen in a way that the time step between two successive lidar samples equals the step size in the turbulence box $x$-axis for a turbulence box with a total duration of $700 \mathrm{~s}$. The resulting spiralshaped pattern is shown in Figure 2, top and bottom right. This scanning pattern is similar to the ZephIR300 commercial lidar. ${ }^{14,15}$

The mapping between the coordinates of the scanning points and the coordinates of the turbulence box grid is carried out by nearest-neighbour interpolation. No turbulence evolution is assumed, and the wind field is assumed to translate towards the rotor at the mean longitudinal wind speed. In the circular pattern described in the preceding texts, the measurement point locations approximately correspond to a radial distance of $85 \%$ of the blade length measured from the hub for the DTU $10 \mathrm{MW}$ reference wind turbine with $178.3 \mathrm{~m}$ rotor diameter. ${ }^{16}$ For the pulsed-lidar pattern, the groups of points corresponding to the multiple ranges from a single laser beam have a uniform spacing and span radial distances between 28 and $85 \%$ of the blade length.

The majority of equations and descriptions given in Sections 2.1 and 2.2 consider the case of constraining a single time series component, e.g. the longitudinal turbulence component, $u$. In a case where additional velocity components need to be constrained, some modifications to the assembled cross-correlation matrices and constraint equations are necessary. In particular, constraining all three components of a turbulence field created using the Mann spectrum requires assembling the following matrices:

$$
\zeta=\left[\begin{array}{ccc}
\zeta_{\mathrm{uu}} & \zeta_{\mathrm{uw}} & \mathbf{0} \\
\zeta_{\mathrm{uw}} & \zeta_{\mathrm{ww}} & \mathbf{0} \\
\mathbf{0} & \mathbf{0} & \zeta_{\mathrm{vv}}
\end{array}\right] ; \quad \mathbf{Z}=\left[\begin{array}{ccc}
\mathbf{Z}_{\mathrm{uu}} & \mathbf{Z}_{\mathbf{u w}} & \mathbf{0} \\
\mathbf{Z}_{\mathbf{u w}}^{T} & \mathbf{Z}_{\mathbf{w w}} & \mathbf{0} \\
\mathbf{0} & \mathbf{0} & \mathbf{Z}_{\mathbf{v v}}
\end{array}\right] ; \quad \mathbf{H}-\tilde{\mathbf{g}}_{\mathbf{c}}=\left[\begin{array}{c}
\mathbf{H}_{\mathbf{u}}-\tilde{\mathbf{g}}_{\mathbf{c}, \mathbf{u}} \\
\mathbf{H}_{\mathbf{w}}-\tilde{\mathbf{g}}_{\mathbf{c}, \mathbf{w}} \\
\mathbf{H}_{\mathbf{v}}-\tilde{\mathbf{g}}_{\mathbf{c}, \mathbf{v}}
\end{array}\right]
$$

where $\zeta_{\mathbf{m n}}, m, n=u, v, w$ are matrices containing the cross correlations between the field and the constraints corresponding to the respective cross-spectral component. The dimension of $\zeta_{\mathrm{mn}}$ is $N \times M$, where $N$ is the total number of points in the field, and $M$ is the number of constraints. $\mathbf{H}_{\mathbf{m}}$ and $\tilde{\mathbf{g}}_{\mathbf{c}, \mathbf{m}}$ are respectively the constraints and the contemporaneous values of the unconstrained field for velocity component $m . \mathbf{Z}_{\mathbf{m n}}$ is a matrix with cross correlations between constraints and has a rank of $M \times M$, and $\mathbf{0}$ is a zero-matrix with the same rank. The presence of the zero matrices is because the Mann model lacks a definition of the $u v$ and $v w$ cross spectra. When constraining turbulence boxes in all three components, this can be exploited by only constraining the $u$ and $w$ components simultaneously, which reduces the sizes of the matrices necessary for the computations.

\subsection{Spectral properties of turbulence}

The statistical properties of the turbulence fields used in the present study are based on Mann's uniform shear spectral model. ${ }^{2,3}$ The Mann model uses an isotropic von Karman turbulence energy spectrum, which is modified to account for the shear deformation and dissipation of the turbulence eddies caused by interaction with the ground surface. The model has three parameters: $\Gamma$ is a non-dimensional number parameterizing the eddy lifetime; $L$ is a length scale describing the size of the most energy-containing eddies; $\alpha \epsilon^{2 / 3}$ is a measure of the energy dissipation. The spectral properties are defined in terms of a spectral velocity tensor, $\Phi_{\mathrm{ij}}(\mathbf{k}),{ }^{17}$ which is a function of the three parameters, $\Gamma, L$ and $\alpha \epsilon^{2 / 3}$, and the wave 
numbers in three dimensions, $\mathbf{k}=\left[k_{1}, k_{2}, k_{3}\right]$. The cross-spectra $\chi_{\mathrm{ij}}, i=1 \ldots 3, j=1 \ldots 3$ are obtained by integrating the spectral tensor over the wave numbers in transverse directions $k_{2}$ and $k_{3}$ :

$$
\chi_{\mathrm{ij}}\left(k_{1}, \Delta_{\mathrm{y}}, \Delta_{\mathrm{z}}\right)=\int \Phi_{\mathrm{ij}}(\mathbf{k}) \exp \left(i\left(k_{2} \Delta_{\mathrm{y}}+k_{3} \Delta_{\mathrm{z}}\right)\right) d \mathbf{k}_{\perp}
$$

where $\int d \mathbf{k}_{\perp}=\int_{-\infty}^{\infty} d k_{2} d k_{3}$, and $\Delta_{\mathrm{y}}$ and $\Delta_{\mathrm{z}}$ are spatial separations respectively along the horizontal and vertical axes $y$ and $z$ which are perpendicular to the wind direction.

The auto- and cross-correlation structure of the turbulence field in the $u$ direction can be derived by inverse Fouriertransforming the $u-u$ cross spectrum $\chi_{11}\left(k_{1}, \Delta_{\mathrm{y}}, \Delta_{z}\right)$ :

$$
R_{\mathrm{uu}}\left(\Delta_{\mathrm{x}}, \Delta_{\mathrm{y}}, \Delta_{\mathrm{z}}\right) \propto \int_{-\infty}^{\infty} \chi_{11}\left(k_{1}, \Delta_{\mathrm{y}}, \Delta_{\mathrm{z}}\right) e^{i k_{1} \Delta x} d k_{1}
$$

The correlation quantities needed for constructing a constrained turbulence box, $\zeta(\mathbf{r})$ and $\mathbf{Z}$, can be easily obtained by evaluating Equation 7, where the distance between two points $(i, j)$ is given as $\mathbf{r}_{\mathrm{ij}}=\left\{\left|x_{\mathrm{j}}-x_{\mathrm{i}}\right|,\left|y_{\mathrm{j}}-y_{\mathrm{i}}\right|,\left|z_{\mathrm{j}}-z_{\mathrm{i}}\right|\right\}$. Because the average wind speed $\bar{u}$ is defined to be homogeneous over the entire turbulence box, the distance along the wind direction $\Delta_{\mathrm{x}}$ can also be represented as a time separation, i.e. $\Delta \mathrm{t}=\left|t_{\mathrm{i}}-t_{\mathrm{j}}\right|$, where $t_{\mathrm{i}}=x_{\mathrm{i}} / \bar{u}$. Because of the finite size of a turbulence box, the integration limits in Equation 7 will in practice be finite and will reflect the range of wave numbers which can exist within a turbulence box based on its size and length of the time step. The correlation matrix is dense, and for large separations, the correlation values are small but not zero, especially at longer turbulence length scales. Therefore, in order to obtain an accurate correlation matrix, it is important that the turbulence spectrum is evaluated as accurately as possible and integrated over the correct frequency range. Figure 3 plots snapshots of the turbulence field in the $y-z$ plane taken at the same $x$ coordinate for three different cases: (i) a reference random realization which serves as a 'target', i.e. its values are considered as constraints when constructing a constrained realization, (ii) an unconstrained random realization and (iii) a constrained realization constructed by using the unconstrained realization (ii) as a basis and selecting constraints from realization (i). An example time series with applied constraints is shown on Figure 4, and Figure 5 shows the autocorrelation functions of the time series shown on Figure 4.At the locations in the $y-z$ plane where the constraints are applied, the constrained field corresponds closely to the target field and matches the autocorrelation of the target field. The close resemblance is present at points close to the constraint locations; however, for locations far away from constraints (e.g. the corners of the turbulence box, Figure 3), the constrained field still resembles the unconstrained source field.

\subsection{Assessment of scanning pattern efficiency using a measure of explained variance}

\subsubsection{Explained variance in a constrained turbulence box}

The variance of the residual field at a point $\mathbf{r}_{\mathrm{i}}=\left\{x_{\mathrm{i}}, y_{\mathrm{i}}, z_{\mathrm{i}}\right\}$, as defined in Equation 3 , is calculated as the difference between two terms - the unconstrained field variance $\sigma^{2}$ and the 'explained variance'-which we denote as

$$
\sigma_{\mathrm{E}}^{2}(\mathbf{r})=\zeta(\mathbf{r}) \mathbf{Z}^{-1} \zeta(\mathbf{r})^{T}
$$
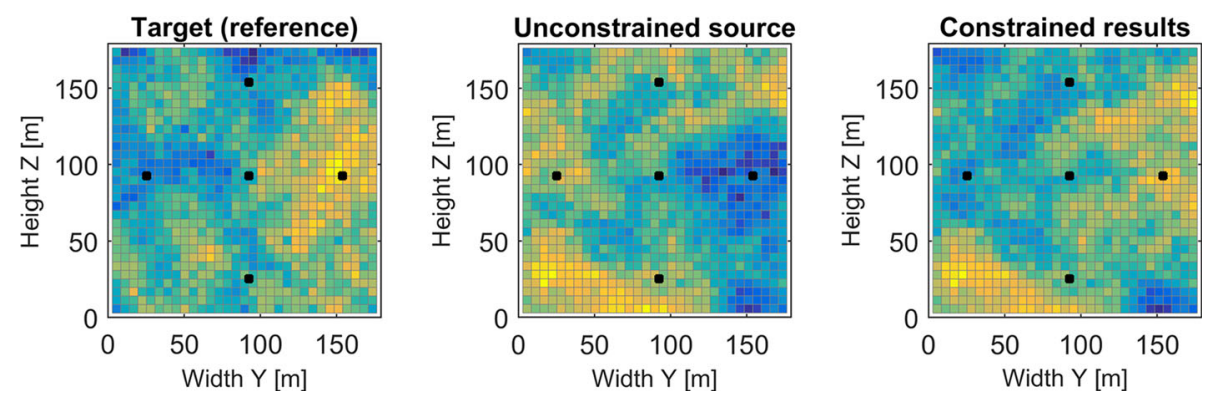

Figure 3. Snapshots of the instantaneous turbulence fields in a $y-z$ plane comparing the field in a constrained turbulence box to the reference field and to the unconstrained field used as a source for the constraint implementation. The dark points indicate the constraint locations in the $y-z$ plane. 


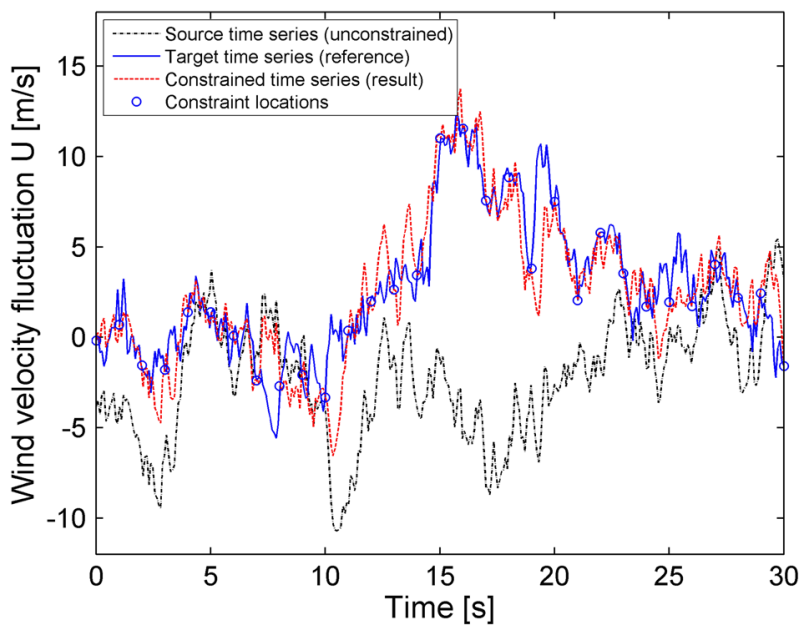

Figure 4. Comparison of constrained and unconstrained time series at a point location where a constraint is applied once per second. Constraint values are shown as blue circles.

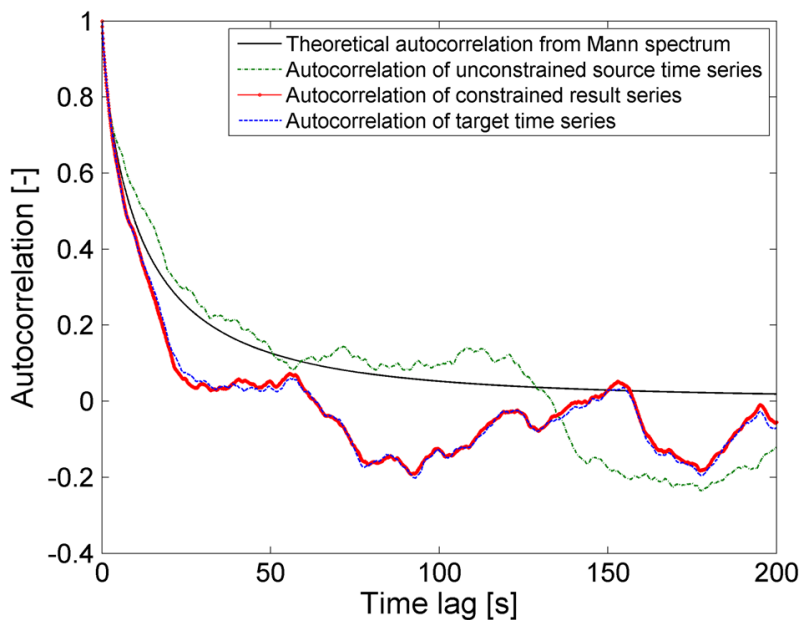

Figure 5. Comparison of autocorrelation functions at a point in the $x-y$ plane where a constraint is applied once per second.

In the preceding texts, the explained variance $\sigma_{\mathrm{E}}^{2}(\mathbf{r})$ is evaluated for the point $\mathbf{r}_{\mathrm{i}}=\left\{x_{\mathrm{i}}, y_{\mathrm{i}}, z_{\mathrm{i}}\right\}$ in the field (as shown earlier, the $x$ coordinate will also represent time as $x_{\mathrm{i}}=t_{\mathrm{i}} \bar{u}$ ). For a field defined in a finite, a three-dimensional domain (e.g. a turbulence box) with dimensions $L_{1}, L_{2}, L_{3}$, the total explained variance can be calculated as the volume integral of the explained variance over the domain:

$$
\overline{\sigma_{\mathrm{E}}^{2}}=\frac{1}{V} \int_{V} \sigma_{\mathrm{E}}^{2}(\mathbf{r}) d \mathbf{r}=\frac{1}{V} \int_{-L_{1} / 2}^{L_{1} / 2} \int_{-L_{2} / 2}^{L_{2} / 2} \int_{-L_{3} / 2}^{L_{3} / 2} \sigma_{\mathrm{E}}^{2}(x, y, z) \cdot d x d y d z
$$

where $V=L_{1} L_{2} L_{3}$ is the volume of the domain.

In the context of constrained random fields, the explained variance is a measure of the proportion of the variance in the random field which is explained by the constraints imposed on it. Because the intended use of constraints in the current study is for representing wind velocities measured by a lidar, the explained variance can be considered as the proportion of the variance in the actually measured field which is transferred to the unconstrained field by imposing constraints. This gives a possibility to assess different scanning patterns with respect to their efficiency in representing the measured turbulence field. 


\subsubsection{Weighting of the explained variance}

The wind field in a turbulence box and the loads and energy production of the wind turbine are coupled through the interaction with the aerodynamics of the blades and the control system of the turbine. Therefore, weighting the explained variance with respect to its effects on the aerodynamic forces may result in better assessment of the efficiency of lidar patterns for reducing statistical uncertainty. Here, we discuss two possible approaches which can be used in combination.

The first suggested modification is the so-called 'rotational sampling', where the scanning speed is synchronized with the rotor angular velocity, and the explained variance is weighted according to the distance between the constraints and the actual position of the blades. Similar considerations have been used by e.g. Simley et al., ${ }^{12}$ where it is shown that rotational sampling provides higher coherence between lidar measurements and the blade effective wind speeds. However, with the present approach to load simulations, turbulence fields are generated independently of the simulation code, meaning that the rotor angular velocity and position are not known at the time of turbulence box generation. This makes the implementation of true rotational sampling matched to actual blade positions infeasible. Instead, the following approximate procedure is implemented: We assume that the turbulence box moves with a given average wind speed $\bar{u}$ towards a hypothetical threebladed rotor with a radius $R$, hub located at coordinates $\left(y_{\text {hub }}, z_{\text {hub }}\right)$ and where the rotor has a mean angular velocity of $\bar{\omega}$ and an initial azimuth $\varphi$. At any given moment $t$, the 'blade-specific explained variance' for a radial position $r$ along the length of one of the blades of the rotor can be estimated by interpolating over the explained variance for the $y-z$ (rotor) plane corresponding to $t$ :

$$
\sigma_{\mathrm{E}}^{2}(t, r)=\sigma_{\mathrm{E}}^{2}\left(x=t \bar{u}, y=y_{\text {hub }}+r \sin (\bar{\omega} t+\varphi), z=z_{\text {hub }}+r \cos (\bar{\omega} t+\varphi)\right)
$$

Then an overall modified explained variance $\overline{\sigma_{\mathrm{R}}^{2}}$ is found by integrating over the blade length and over time:

$$
\overline{\sigma_{\mathrm{R}}^{2}}=\frac{1}{T} \cdot \frac{1}{R} \int_{0}^{t} \int_{0}^{R} \sigma_{\mathrm{E}}^{2}(t, r) d t d r
$$

Because the motion of scanning patterns is not synchronized with the rotor speed, the result of Equation 11 will depend on the initial conditions. To account for that, the rotationally sampled explained variance is calculated for a number of rotor initial positions $\varphi$ ranging from 0 to $2 \pi$, and the final result for $\overline{\sigma_{\mathrm{R}}^{2}}$ is taken as the average from all initial positions.

Further modification of the explained variance is applied by weighting according to the aerodynamic force distribution over the blade. The left plot in Figure 6 shows the local thrust coefficient as a function of radial distance from the hub centre. Integrated over the blade length, the local thrust coefficient can be used to determine the overall thrust force $T$ per blade and approximate the blade root out of plane moment $M_{\mathrm{x}}$ :

$$
\begin{aligned}
& T \propto \int_{0}^{R} C_{\mathrm{T}, \mathrm{L}}(r) d r \\
& M_{\mathrm{X}} \propto \int_{0}^{R} C_{\mathrm{T}, \mathrm{L}}(r) \cdot r d r
\end{aligned}
$$
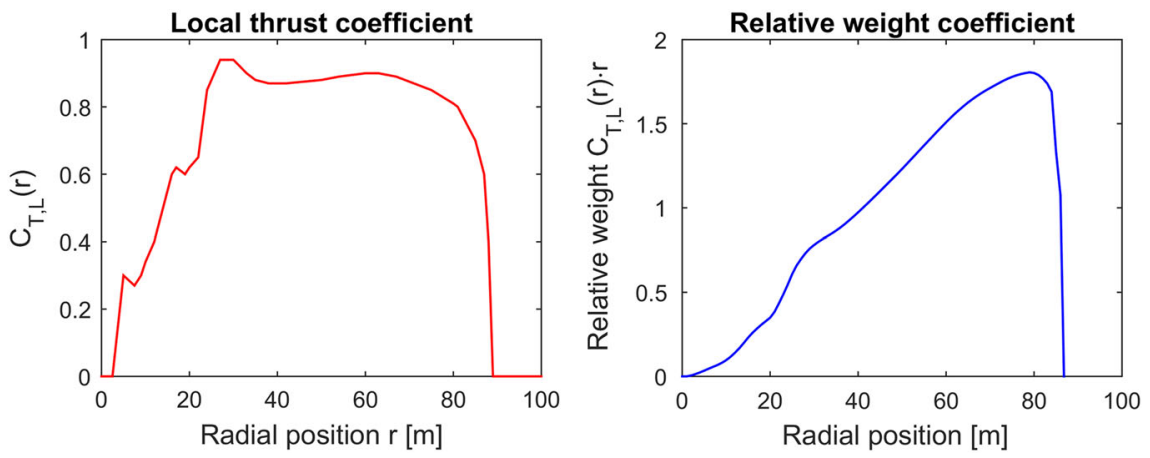

Figure 6. Left: local thrust coefficient as function of blade radial position at $9 \mathrm{~m} / \mathrm{s}$ average wind speed. Right: relative weight of variance as function of distance from hub centre. 
where $C_{\mathrm{T}, \mathrm{L}}(r)$ is the local thrust coefficient at radial position $r$. Therefore, the integrand in the second line of Equation 12 can be considered as the contribution of the aerodynamic force at a given blade radial position to the root out of plane bending moment:

$$
w(r)=C_{\mathrm{T}, \mathrm{L}}(r) \cdot r
$$

The weighting function, normalized to have a unity mean, is shown on the right side of Figure 6. For any points falling outside the rotor plane, $w(r)$ is considered equal to 0 . The weighted explained variance $\overline{\sigma_{\mathrm{W}}^{2}}$ is calculated by multiplying $w(r)$ with $\sigma_{\mathrm{E}}^{2}(t, r)$ from Equation 11:

$$
\overline{\sigma_{\mathrm{W}}^{2}}=\frac{1}{T} \cdot \frac{1}{R} \int_{0}^{t} \int_{0}^{R} w(r) \sigma_{\mathrm{E}}^{2}(t, r) d t d r
$$

\subsubsection{Explained variance of lidar scanning patterns}

We select 12 example patterns which are applicable to the current lidar technology (Figure 7) and estimate the explained variance achieved for different pattern sizes and scanning periods. For faster calculations, the estimation is carried out over $1 / 8$ of the turbulence box length, i.e. 1024 points corresponding to $87.5 \mathrm{~s}$. For the sake of obtaining a valid comparison in all the studied cases, the number of constraints applied to a single turbulence box is kept as close to 1024 as possible. For patterns where only one measurement is taken at a time (e.g. patterns resembling continuous-wave lidar scanning), this is achieved with a sampling interval equal to the time step of the turbulence box which results in one measurement per time step. For scanning patterns with multiple simultaneous measurements, the measurement interval is taken as the multiple of the time step in the turbulence box which will result in a number of constraints as close to 1024 as possible.

The first four pattern examples mimic measurements with one or more simultaneously measuring beams pointing at a fixed location forward and measuring at a single range. In order to keep the same total number of constraints, the sampling frequency is lower for configurations with a larger number of simultaneously measuring beams. The fifth pattern example resembles the scanning behaviour of a pulsed lidar with five beams, where each of the beams points in a fixed direction and measures in 10 line-of-sight distance ranges simultaneously. The distance between any two measurement ranges is taken as
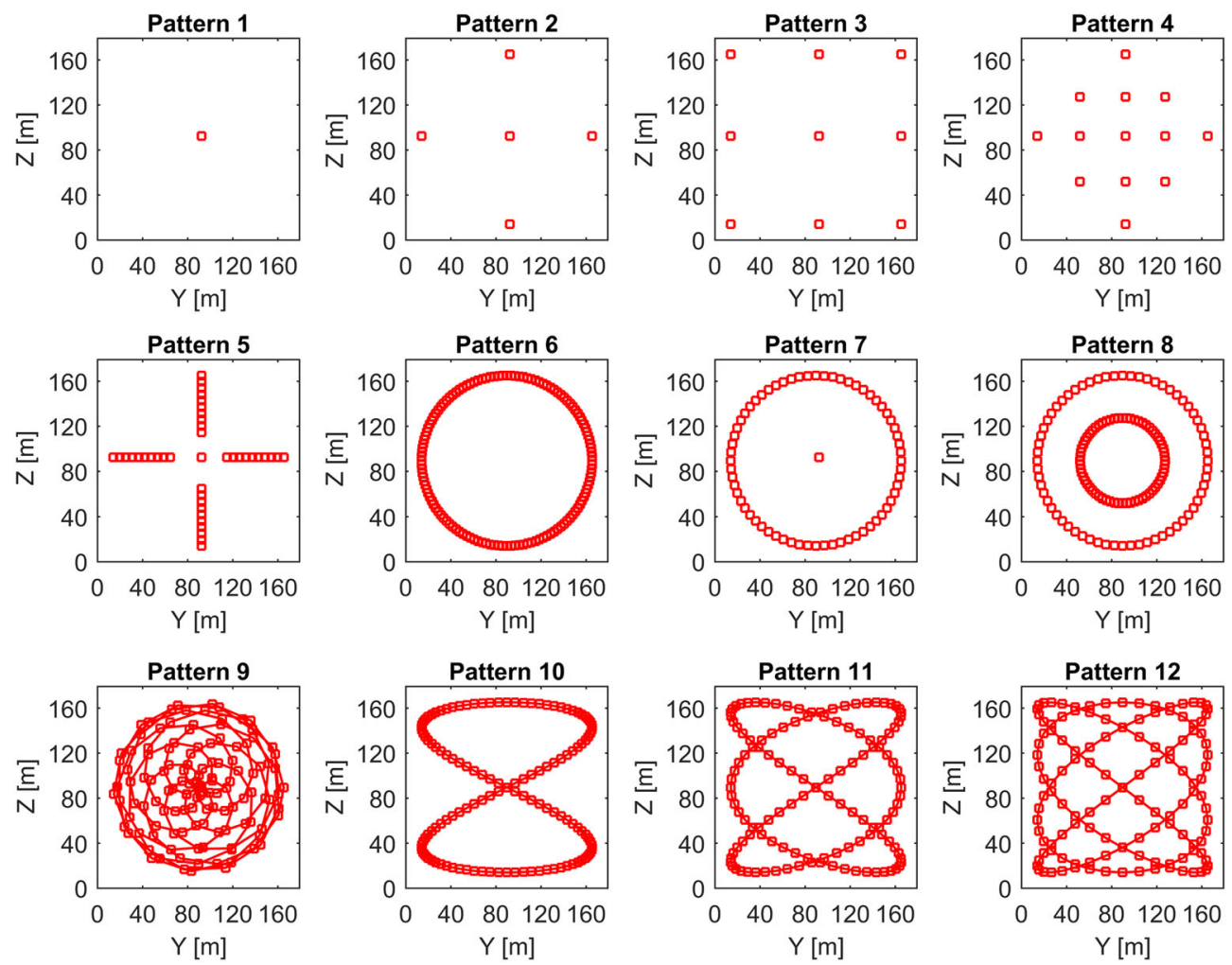

Figure 7. Various lidar scanning patterns assessed by the explained variance achieved when using the measurements as constraints. 
the distance which the mean wind field passes in $1 \mathrm{~s}$. The measurements are taken with one beam at a time. The remaining pattern examples assume that there are one or two beams which are in a continuous revolving motion and draw a circular pattern (patterns 6 to 8), a combination of two circular patterns with non-coincident centres of rotation (pattern 9) resembling the movement of a rotating spinner lidar, ${ }^{18}$ or a Lissajous curve (patterns 10 to 12). ${ }^{19}$ This continuous scanning behaviour is characteristic for the continuous-wave lidar technology. For moving beams, the term 'pattern period' defines the time needed for a beam to complete one full cycle of the pattern. Table I gives a more detailed overview of the scanning patterns including the pattern parameters.

Using the expressions in Equations 8 and 9, the explained variance $\overline{\sigma_{\mathrm{E}}^{2}}$ associated with each of the 12 patterns described in Table I is calculated. For all patterns except pattern 1, nine pattern sizes are analysed ranging from 0.49 to 1 rotor diameters in size increments of $5.6 \mathrm{~m}$ ( 0.063 rotor diameters). For patterns 6 to 12, four analyses with different pattern periods (1.37 to $10.9 \mathrm{~s}$ ) are carried out at each pattern size. Identical calculations are also carried out for the rotationally sampled, weighted explained variance $\overline{\sigma_{\mathrm{W}}^{2}}$ as given by Equation 11. Figure 8 shows the maximum obtained values of explained variance per pattern type for all pattern sizes and periods analysed. The results are normalized with respect to the actual variance in the turbulence box, then $\overline{\sigma_{\mathrm{E}}^{2}}=1$ means that the entire variance in the domain is explained by the constraints, while $\overline{\sigma_{\mathrm{E}}^{2}}=0$ means none of the actual variance is explained. The results show that constraints based on fixed-point patterns with nine or more points, as well as moving-scan patterns, are capable of achieving above $80 \%$ explained variance over a reference period of $90 \mathrm{~s}$. When considering a longer period of e.g. $700 \mathrm{~s}$, the explained variance will increase further as there will be some additional gains over the low-frequency part of the spectrum.

Rotational sampling of the explained variance gives more weight to points close to the centre of rotation, while the weighting according to the relative bending moment has an opposite effect. The overall result is that the rotationally sampled, weighted explained variance $\overline{\sigma_{\mathrm{W}}^{2}}$ is slightly higher than the non-weighted $\overline{\sigma_{\mathrm{E}}^{2}}$, but the variation of explained variance from pattern to pattern remains similar. The effect of weighting the explained variance is more pronounced on the optimal pattern size which is shown on Figure 9. The optimal pattern size (i.e. the pattern size which results in the highest explained variance) is slightly smaller for the weighted cases. For example, for the patterns following a Lissajous curve, the highest weighted explained variance is achieved at approximately 0.81 rotor diameters compared with 0.88 rotor diameters for the non-weighted case.

The performance of different patterns is influenced both by the pattern size and pattern period. For all pattern types, the general tendency is that bigger pattern sizes result in more explained variance, while a pattern period of about $5.5 \mathrm{~s}$ seems to be optimal. Figure 10 shows an example with the results as function of pattern size and period for pattern 11 .

Table I. Overview of the patterns used in the assessment of explained variance.

\begin{tabular}{|c|c|c|c|c|c|c|}
\hline $\begin{array}{l}\text { Pattern } \\
\text { number }\end{array}$ & Description & $\begin{array}{l}\text { Points } \\
\text { per sample }\end{array}$ & $\begin{array}{l}\text { Sampling } \\
\text { frequency }[\mathrm{Hz}]\end{array}$ & $\begin{array}{c}\text { Pattern } \\
\text { periods [s] }\end{array}$ & $\begin{array}{l}\text { Pattern sizes in } \\
\text { rotor diameters }\end{array}$ & $\begin{array}{c}\text { Total } \\
\text { constraints }\end{array}$ \\
\hline 1 & Single point at hub location & 1 & 11.7 & - & - & 1024 \\
\hline 2 & $\begin{array}{l}\text { Four points on the vertices of a square } \\
\text { and a central point }\end{array}$ & 5 & 2.34 & - & 0.49 to 1 & 1020 \\
\hline 3 & $\begin{array}{l}\text { Nine points arranged on the vertices, } \\
\text { mid-sides and the centre of a square }\end{array}$ & 9 & 1.3 & - & 0.49 to 1 & 1017 \\
\hline 4 & 13 points arranged in a square pattern & 13 & 0.9 & - & 0.49 to 1 & 1014 \\
\hline 5 & $\begin{array}{l}\text { Five beams with the same configuration as } \\
\text { in pattern } 2 \text {, scanning in } 10 \text { ranges each, } \\
\text { one beam at a time }\end{array}$ & 10 & 1.17 & - & 0.49 to 1 & 1020 \\
\hline 6 & Single-beam scanning a circular pattern & 1 & 11.7 & 1.37 to 10.9 & 0.49 to 1 & 1024 \\
\hline 7 & $\begin{array}{l}\text { One beam scanning a circular pattern and } \\
\text { a stationary forward-pointing beam }\end{array}$ & 2 & 5.85 & 1.37 to 10.9 & 0.49 to 1 & 1024 \\
\hline 8 & $\begin{array}{l}\text { Two concentric circles, rotation is in the } \\
\text { same direction. }\end{array}$ & 2 & 5.85 & 1.37 to 10.9 & 0.49 to 1 & 1024 \\
\hline 9 & $\begin{array}{l}\text { A single beam following the path drawn } \\
\text { by the combination of two eccentric } \\
\text { circular patterns with different rotation periods }\end{array}$ & 2 & 11.7 & 1.37 to 10.9 & 0.49 to 1 & 1024 \\
\hline 10 & Lissajous figure with parameters $a=2$ and $b=1$ & 1 & 11.7 & 1.37 to 10.9 & 0.49 to 1 & 1024 \\
\hline 11 & Lissajous figure with parameters $a=3$ and $b=2$ & 1 & 11.7 & 1.37 to 10.9 & 0.49 to 1 & 1024 \\
\hline 12 & Lissajous figure with parameters $a=4$ and $b=3$ & 1 & 11.7 & 1.37 to 10.9 & 0.49 to 1 & 1024 \\
\hline
\end{tabular}




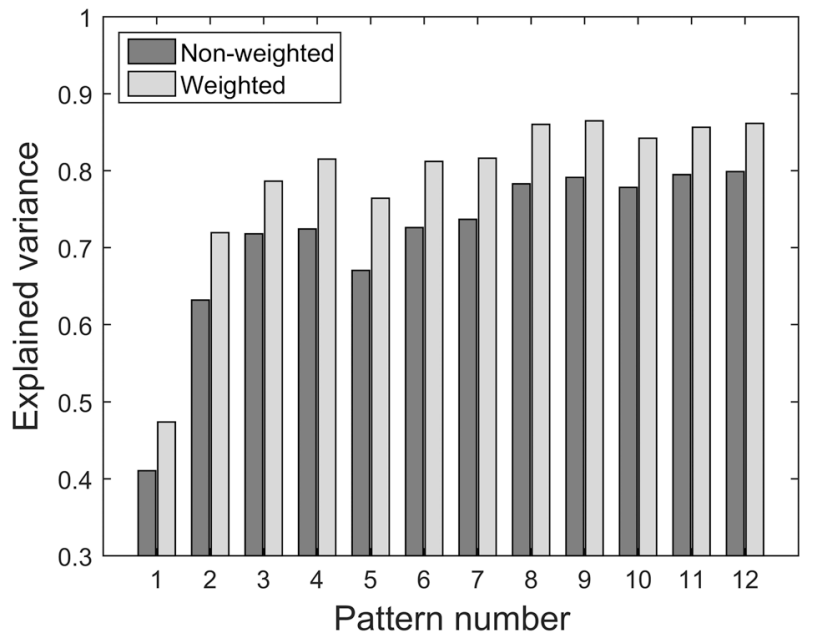

Figure 8. Maximum achieved values for the total explained variance $\mu_{\sigma_{\mathrm{E}}^{2}}$ and the rotationally sampled, weighted explained variance $\sigma_{\mathrm{W}}^{2}$ as a function of pattern type. For each pattern type, the value shown is the highest value of the total explained variance achieved over all pattern periods and sizes.

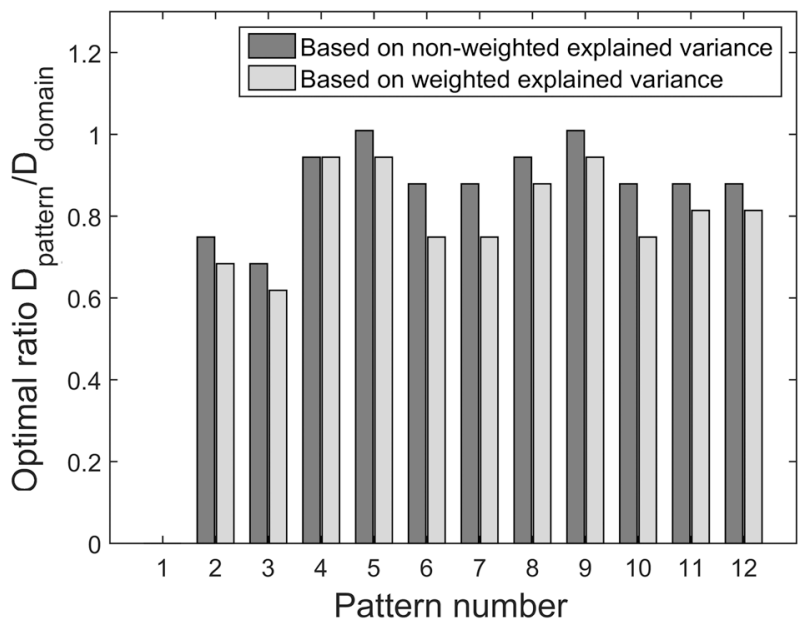

Figure 9. Optimal pattern sizes relative to the entire domain size, where the domain size approximately equals the wind turbine rotor diameter. For any pattern type, the optimal size is determined as the size which results in the largest explained variance $\mu_{\sigma_{\mathrm{E}}^{2}}$ and regardless of pattern period.
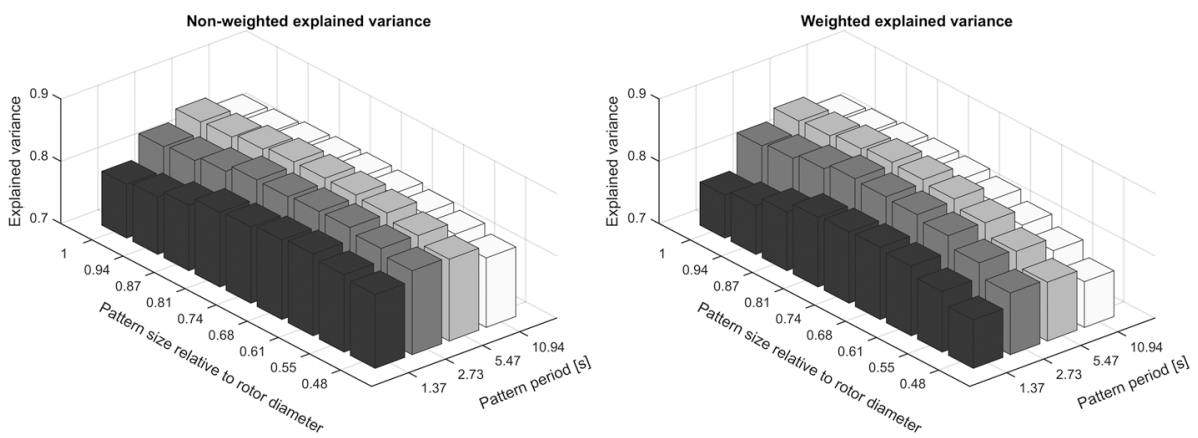

Figure 10. Explained variance as function of pattern size and period, for a single beam scanning a Lissajous figure (Pattern 11). Left: non-weighted explained variance $\left(\mu_{\sigma_{\mathrm{E}}^{2}}\right)$. Right: explained variance $\left(\sigma_{W}^{2}\right)$, rotationally sampled and weighted according to the aerodynamic force distribution over the blade. 


\section{CONSTRAINED TURBULENCE FIELD AND LOAD UNCERTAINTY}

In the previous section, we demonstrated a method for constructing constrained turbulence fields and showed how this method can be used to incorporate lidar measurement data in the turbulence boxes used for wind turbine load simulations. In the following, we present a numerical study assessing the possible effect of incorporating lidar measurement data on load uncertainty.

\subsection{Load simulations}

For the purpose of the study, we carry out dynamic load simulations using the aeroelastic code Hawc $2 .{ }^{20}$ The wind turbine used the DTU $10 \mathrm{MW}$ reference wind turbine as an example. ${ }^{16}$ The simulation conditions are based on the normal production load case DLC1.1 and external condition class IEC 1A as defined by the IEC61400-1, ed.3 standard. ${ }^{21}$ For each study case, we carry out 18 simulations per mean wind speed, for mean wind speeds between 4 and $25 \mathrm{~m} / \mathrm{s}$ in $1 \mathrm{~m} / \mathrm{s}$ bins, resulting in 396 simulations in each studied constrained simulation. The relatively large number of seeds per wind speed (for comparison, the IEC 61400-1 requires 15 seeds per wind speed) gives better possibilities for evaluating the statistical variation in the loads. Each simulation in a study case uses a unique turbulence field defined by specifying different start seed number for the computer's random number generator. We make use of two sets of turbulence seeds, which we denote as $\boldsymbol{A}=\left[a_{1}, a_{2}\right.$, $\left.\ldots, a_{396}\right]$ and $\boldsymbol{B}=\left[b_{1}, b_{2}, \ldots, b_{396}\right]$. A total of 12 simulation sets are defined corresponding to 12 study cases:

1. A 'target' reference case with simulations using unconstrained turbulence boxes from the turbulence seed set $\boldsymbol{A}$.

2. A baseline case with simulations using unconstrained turbulence boxes from set $\boldsymbol{B}$.

3. to 9) Constrained cases where point values from turbulence set $\boldsymbol{A}$ are incorporated as constraints to the source turbulence box set $\boldsymbol{B}$. The constraints from set $\boldsymbol{A}$ are defined at locations corresponding to different lidar patterns as follows, with pattern numbers corresponding to the ones shown on Figure 7:

4. Pattern 1: Single forward-pointing beam, with a scanning frequency of $1 \mathrm{~Hz}$, which is approximately the $6 \mathrm{P}$ frequency at rated speed of the turbine (700 constraints per turbulence box). The resulting turbulence fields are denoted as set $\boldsymbol{C}$.

5. Pattern 2: Five-beam pattern with a maximum distance between the points 0.7 rotor diameters, scanning frequency of $1 \mathrm{~Hz}$ (3500 constraints per turbulence box). Denoted as set $\boldsymbol{D}$.

6. Pattern 5: Pattern with five beams measuring in sequential order, each beam measuring simultaneously at 10 different ranges along the line of sight. Pattern size is 0.87 rotor diameters, and the scanning frequency is $0.2 \mathrm{~Hz}$ per beam, i.e. $1 \mathrm{~Hz}$ overall (8080 constraints per turbulence box). Denoted as set $\boldsymbol{E}$.

7. Pattern 6: Circular pattern with a pattern diameter equal to 0.7 rotor diameters, a scanning frequency of $11.7 \mathrm{~Hz}$ and rotation period of $2.73 \mathrm{~s}$ (8192 constraints per turbulence box). Denoted as set $\boldsymbol{F}$.

8. Pattern 11: Pattern following a Lissajous curve with parameters (3,2), a pattern size equal to 0.87 rotor diameters, a scanning frequency of $11.7 \mathrm{~Hz}$ and a rotation period of $5.46 \mathrm{~s}$ (8192 constraints per turbulence box). Denoted as set G.

9. Pattern 9: Double co-rotating pattern with a size equal to 0.87 rotor diameters, a scanning frequency of $11.7 \mathrm{~Hz}$ and a rotation period of $5.46 \mathrm{~s}$ (8192 constraints per turbulence box). Denoted as set $\boldsymbol{H}$.

10. to 12): Using the same pattern characteristics as case $H$, several perturbations are applied in order to test the sensitivity of the results to changes in inputs:

11. Pattern 9, both $u$ and $v$ components constrained: The same pattern characteristics as set $H$; however, both the $u$ and $v$ components in the turbulence box are constrained. Denoted as set $\boldsymbol{H} \mathbf{1}$.

12. Pattern 9, with $u, v$ and $w$ constrained: Same pattern as set $H$, with all three turbulence components constrained. Denoted as set $\boldsymbol{H} \mathbf{2}$.

13. Pattern 9, doubled amount of constraints: Same pattern as set $H$, however, with double frequency $(23.4 \mathrm{~Hz})$ which results in twice the amount of constraints as in case $H$. Denoted as set $\boldsymbol{H 3}$.

14. Pattern 9, half the amount of constraints: Same pattern as set $H$, however, with half the frequency $(5.85 \mathrm{~Hz})$ which results in half the amount of constraints as in case $H$. Denoted as set $\boldsymbol{H 4}$.

The choice of two of the constraint patterns (cases $E$ and $F$ ) is a result of the two patterns actually being considered or already implemented by lidar manufacturers. ${ }^{14,15}$ The other cases are chosen to represent one pattern from each of the pattern families considered in Section 2.4. The perturbation cases are intended as an assessment to whether the currently chosen modelling approach is detailed enough. For instance, the recovery of all three wind field components from lineof-sight lidar measurements may be a significant challenge. It is therefore important to assess to what extent all three components are necessary, and whether using only the longitudinal component can be sufficient for load validation purposes.

The turbulence length scale affects the correlation length and thus the distance around a constraint location where the field is affected by the constraint. Considering this, the setup described in the preceding texts was used in two separate sets 
of simulations, one using a Mann turbulence model with length scale parameter of $L=29.4 \mathrm{~m}$, and another with $L=72 \mathrm{~m}$. The former value is recommended in the IEC61400-1 standard, while the latter is chosen based on studies describing the typical values of $L$ under neutrally unstable atmospheric conditions in flat terrains. ${ }^{22}$

\subsection{Uncertainty analysis}

We assess the realization-to-realization uncertainty in the load simulations by calculating the ratios between the quantity of interest (energy production, extreme loads or damage-equivalent fatigue loads) observed in the reference load case (set $\boldsymbol{A}$ ) to the quantity observed in the simulations from the remaining cases. One ratio is calculated for each turbulence seed number, leading to uncertainty variables defined as e.g.

$$
X_{\mathrm{B}}(i)=\frac{F\left(b_{\mathrm{i}}\right)}{F\left(a_{\mathrm{i}}\right)}
$$

where $i$ is the seed number, $F($.$) is a function of the load time series (e.g. maximum load, damage-equivalent fatigue load,$ DEL, or energy production), $a_{i}$ and $b_{i}$ are the turbulence seeds from sets $\boldsymbol{A}$ and $\boldsymbol{B}$ respectively, and $X_{B}$ is a variable characterizing the uncertainty in simulations with turbulence set $\boldsymbol{B}$ with respect to the reference simulation set $\boldsymbol{A}$. The assessment of the uncertainty is then based on the statistics (mean and variance) of the uncertainty variables defined in the preceding texts. Table II lists the standard deviations of the uncertainty variables with respect to the energy production, where the energy production is defined as the integral of the electrical power over one simulation period (10 min). Only wind speeds from 4 to $14 \mathrm{~m} / \mathrm{s}$ are considered because for wind speeds above the turbine's rated speed $(11.4 \mathrm{~m} / \mathrm{s}$ for steady, non-turbulent wind), the uncertainty in the energy production tends to zero. Figure 11 shows an example of the empirically determined probability density functions of the power production uncertainty for turbulence length scale $L=72$. In general, smaller standard deviation of the uncertainty results in a narrower distribution and a more pronounced peak in the probability density function, as seen e.g. cases $E, G$ and $H$ in Figure 11 .

Table III lists the sample standard deviations of the uncertainty variables regarding the extremes of six load channels, and the same result is shown graphically on Figure 12. Table IV compares the uncertainty in damage-equivalent fatigue

Table II. Standard deviations of uncertainty variables regarding energy production estimated from simulations, normalized with respect to the standard deviation for unconstrained turbulence boxes.

\begin{tabular}{lcccccccc}
\hline & $L$ & $\frac{\operatorname{Std}\left(X_{\mathrm{B}}\right)}{\operatorname{Std}\left(X_{\mathrm{B}}\right)}$ & $\frac{\operatorname{Std}\left(X_{\mathrm{C}}\right)}{\operatorname{Std}\left(X_{\mathrm{B}}\right)}$ & $\frac{\operatorname{Std}\left(X_{\mathrm{D}}\right)}{\operatorname{Std}\left(X_{\mathrm{B}}\right)}$ & $\frac{\operatorname{Std}\left(X_{\mathrm{B}}\right)}{\operatorname{Std}\left(X_{\mathrm{B}}\right)}$ & $\frac{\operatorname{Std}\left(X_{\mathrm{F}}\right)}{\operatorname{Std}\left(X_{\mathrm{B}}\right)}$ & $\frac{\operatorname{Std}\left(X_{\mathrm{G}}\right)}{\operatorname{Std}\left(X_{\mathrm{B}}\right)}$ & $\frac{\operatorname{Std}\left(X_{\mathrm{H}}\right)}{\operatorname{Std}\left(X_{\mathrm{B}}\right)}$ \\
\cline { 2 - 8 } Units & $m$ & $\%$ & $\%$ & $\%$ & $\%$ & $\%$ & $\%$ & $\%$ \\
\hline Energy produced & 29.4 & 100 & 76 & 32 & 23 & 97 & 20 & 12 \\
& 72 & 100 & 93 & 25 & 22 & 76 & 16 & 10 \\
\hline
\end{tabular}

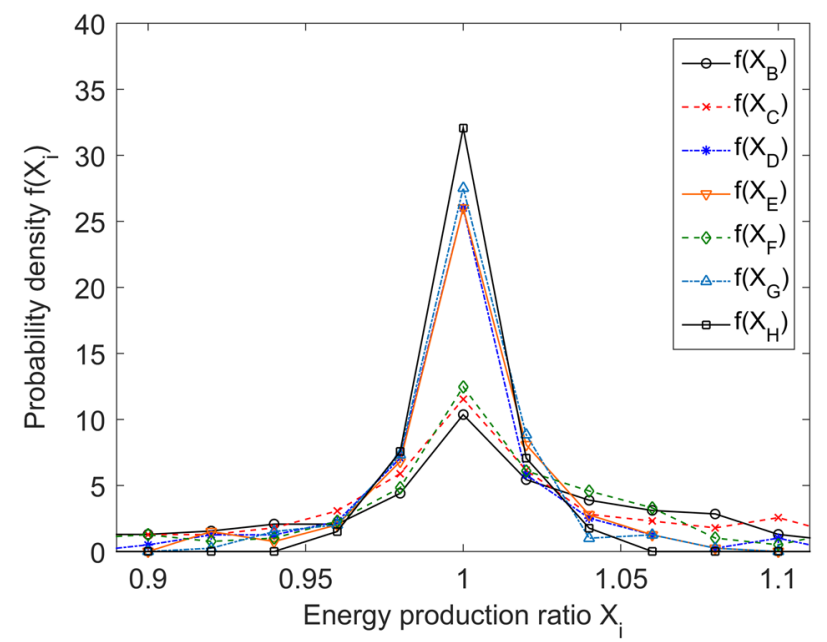

Figure 11. Observed probability densities of the ratios representing the realization-to-realization uncertainty in energy production. 
Table III. Standard deviations of uncertainty variables regarding absolute extreme values observed in simulations, normalized with respect to the standard deviation for unconstrained turbulence boxes.

\begin{tabular}{|c|c|c|c|c|c|c|c|c|}
\hline Channel & $L$ & $\frac{\operatorname{Std}\left(X_{\mathrm{B}}\right)}{\operatorname{Std}\left(X_{\mathrm{B}}\right)}$ & $\frac{\operatorname{Std}\left(X_{\mathrm{C}}\right)}{\operatorname{Std}\left(X_{\mathrm{B}}\right)}$ & $\frac{\operatorname{Std}\left(X_{\mathrm{D}}\right)}{\operatorname{Std}\left(X_{\mathrm{B}}\right)}$ & $\frac{\operatorname{Std}\left(X_{\mathrm{E}}\right)}{\operatorname{Std}\left(X_{\mathrm{B}}\right)}$ & $\frac{\operatorname{Std}\left(X_{\mathrm{F}}\right)}{\operatorname{Std}\left(X_{\mathrm{B}}\right)}$ & $\frac{\operatorname{Std}\left(X_{\mathrm{G}}\right)}{\operatorname{Std}\left(X_{\mathrm{B}}\right)}$ & $\frac{\operatorname{Std}\left(X_{\mathrm{H}}\right)}{\operatorname{Std}\left(X_{\mathrm{B}}\right)}$ \\
\hline Units & $m$ & $\%$ & $\%$ & $\%$ & $\%$ & $\%$ & $\%$ & $\%$ \\
\hline Tower F-A & 29.4 & 100 & 85 & 80 & 76 & 95 & 64 & 58 \\
\hline Tower S2S & 29.4 & 100 & 99 & 93 & 89 & 100 & 87 & 83 \\
\hline Yaw & 29.4 & 100 & 77 & 61 & 59 & 69 & 46 & 46 \\
\hline Shaft & 29.4 & 100 & 88 & 54 & 41 & 92 & 39 & 29 \\
\hline Blade F & 29.4 & 100 & 87 & 71 & 70 & 93 & 63 & 56 \\
\hline Blade E & 29.4 & 100 & 87 & 75 & 68 & 101 & 64 & 61 \\
\hline Tower F-A & 72 & 100 & 97 & 69 & 71 & 78 & 58 & 56 \\
\hline Tower S2S & 72 & 100 & 88 & 80 & 79 & 86 & 77 & 73 \\
\hline Yaw & 72 & 100 & 70 & 50 & 47 & 52 & 35 & 35 \\
\hline Shaft & 72 & 100 & 100 & 42 & 34 & 70 & 33 & 26 \\
\hline Blade F & 72 & 100 & 97 & 63 & 57 & 70 & 50 & 45 \\
\hline Blade E & 72 & 100 & 105 & 63 & 58 & 61 & 53 & 52 \\
\hline
\end{tabular}
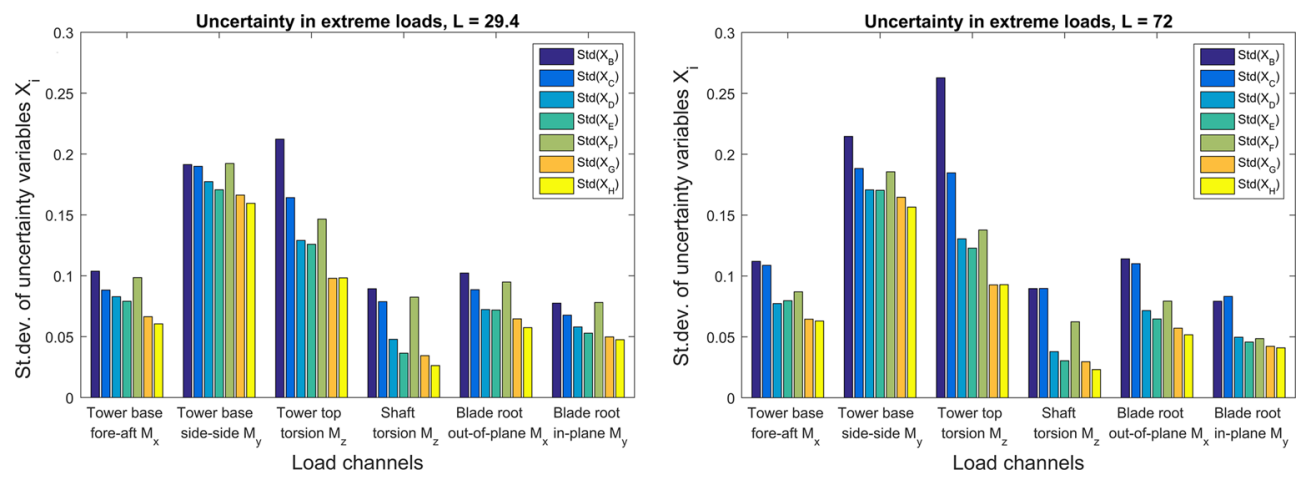

Figure 12. Observed standard deviations of the ratios representing the realization-to-realization uncertainty in extreme loads over mean wind speeds from 4 to $25 \mathrm{~m} / \mathrm{s}$.

Table IV. Standard deviations of uncertainty variables regarding damage-equivalent fatigue loads observed in simulations.

\begin{tabular}{|c|c|c|c|c|c|c|c|c|}
\hline Channel & $L$ & $\frac{\operatorname{Std}\left(X_{\mathrm{B}}\right)}{\operatorname{Std}\left(X_{\mathrm{B}}\right)}$ & $\frac{\operatorname{Std}\left(X_{\mathrm{C}}\right)}{\operatorname{Std}\left(X_{\mathrm{B}}\right)}$ & $\frac{\operatorname{Std}\left(X_{\mathrm{D}}\right)}{\operatorname{Std}\left(X_{\mathrm{B}}\right)}$ & $\frac{\operatorname{Std}\left(X_{\mathrm{E}}\right)}{\operatorname{Std}\left(X_{\mathrm{B}}\right)}$ & $\frac{\operatorname{Std}\left(X_{\mathrm{F}}\right)}{\operatorname{Std}\left(X_{\mathrm{B}}\right)}$ & $\frac{\operatorname{Std}\left(X_{\mathrm{G}}\right)}{\operatorname{Std}\left(X_{\mathrm{B}}\right)}$ & $\frac{\operatorname{Std}\left(X_{\mathrm{H}}\right)}{\operatorname{Std}\left(X_{\mathrm{B}}\right)}$ \\
\hline Units & $m$ & $\%$ & $\%$ & $\%$ & $\%$ & $\%$ & $\%$ & $\%$ \\
\hline Tower F-A & 29.4 & 100 & 63 & 56 & 53 & 87 & 40 & 39 \\
\hline Tower S2S & 29.4 & 100 & 96 & 87 & 84 & 99 & 84 & \\
\hline Yaw & 29.4 & 100 & 41 & 35 & 33 & 79 & 26 & 23 \\
\hline Shaft & 29.4 & 100 & 75 & 47 & 40 & 68 & 28 & 26 \\
\hline Blade F & 29.4 & 100 & 75 & 55 & 51 & 83 & 44 & 41 \\
\hline Blade E & 29.4 & 100 & 75 & 54 & 46 & 149 & 45 & 40 \\
\hline Tower F-A & 72 & 100 & 57 & 42 & 42 & 62 & 30 & 29 \\
\hline Tower S2S & 72 & 100 & 86 & 81 & 80 & 89 & 80 & 81 \\
\hline Yaw & 72 & 100 & 43 & 26 & 25 & 53 & 18 & 17 \\
\hline Shaft & 72 & 100 & 74 & 34 & 27 & 54 & 22 & 20 \\
\hline Blade F & 72 & 100 & 77 & 47 & 44 & 64 & 35 & 34 \\
\hline Blade E & 72 & 100 & 136 & 37 & 34 & 48 & 32 & 30 \\
\hline
\end{tabular}

loads for the same channels, using $S-N$ curve slopes of $m=12$ for blade loads and $m=4$ for all remaining components. These results are also shown on Figure 13. For all variables, the mean values were very close to 1 ; therefore, the mean values are not reported further. For the sake of comparison, all uncertainties from the simulations with constrained turbulence boxes (cases $C$ to $H$ ) are normalized with the baseline uncertainty from unconstrained turbulence simulations $\left(X_{\mathrm{B}}\right)$. 

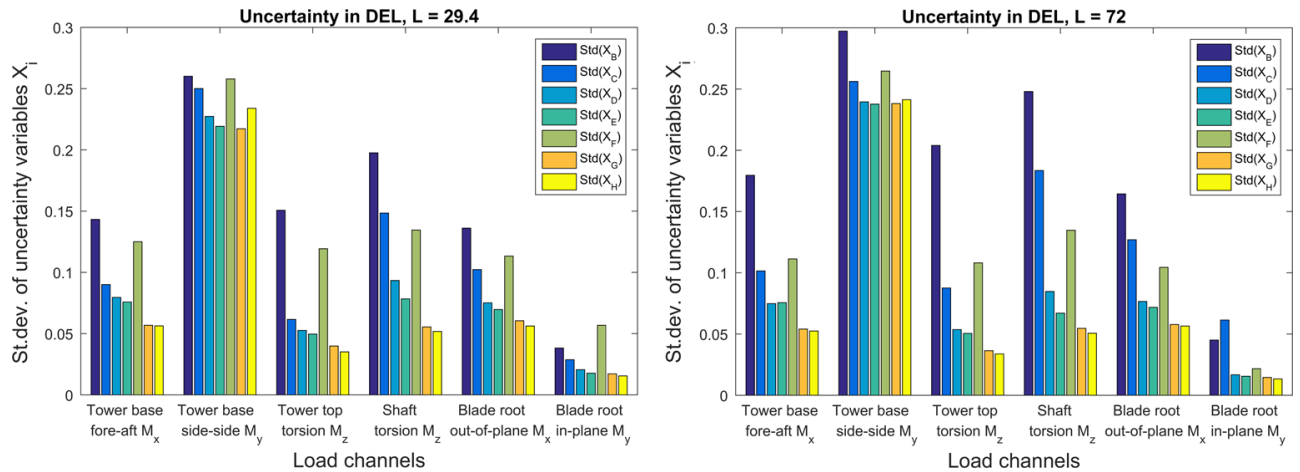

Figure 13. Observed standard deviations of the ratios representing the realization-to-realization uncertainty in damage-equivalent fatigue loads over mean wind speeds from 4 to $25 \mathrm{~m} / \mathrm{s}$.

The load channels considered are (i) tower base fore-aft moment (denoted 'Tower F-A' in the tables), (ii) tower base sideto-side moment (denoted Tower S2S), (iii) the yaw moment at tower top (denoted Yaw), (iv) main shaft torsion (denoted Shaft), (v) blade root flap-wise moment (denoted 'Blade F') and (vi) blade root edge-wise moment (denoted Blade E).

Table $\mathrm{V}$ lists the results from the perturbation cases $H 1$ to $H 4$, with the results from their baseline case $H$ used as reference. Only a few of the results are selected which are indicative of the overall trends in the data.

\subsection{Discussion of results}

The outcomes from the load analysis indicate that applying constraints to turbulence boxes based on simulated lidar wind velocity measurements results in turbulence fields which have stronger similarities to the target fields, which can potentially reduce the load simulation uncertainty. Significant reduction in statistical uncertainty (reduction of standard deviation by a factor between 2 and 5) was observed for wind driven turbine responses such as the energy production as well as the majority of the load components, while non-wind-driven responses such as the tower base side-to-side moment were practically unaffected. The factor of reduction in uncertainty with different scanning patterns tends to be bigger for fatigue damage equivalent loads than for extremes. The overall uncertainty is slightly bigger for a turbulence length scale of $L=72$ compared with $L=29.4$; however, in the constrained cases, this is compensated by a larger reduction in uncertainty. The larger reduction in uncertainty for $L=72$ can be explained by the larger correlation length, which results in a stronger influence of the constraints on the resultant turbulence field. In all comparisons, the moving-beam scanning patterns showed the smallest uncertainty, with the exception of the circular scanning pattern. This is contrary to the findings in Kragh et al., ${ }^{18}$ where a circular scanning pattern showed a superior performance in determining yaw error in comparison to a 2-D scanning pattern similar to the double co-rotating pattern used in the present study. This shows that for different purposes, i.e. yaw error estimation versus load validation, the optimal scanning patterns may be different. Furthermore, the performance of the circular pattern seems to be inferior to the performance of other patterns with similar or even lower explained variance (e.g. the five-beam pattern). Our conclusion is that the explained variance is not the only factor which determines the uncertainty associated with the different scanning patterns—other factors may include the behaviour of the

Table V. Standard deviations of uncertainty variables for simulation cases.

\begin{tabular}{|c|c|c|c|c|c|c|}
\hline Channel & $L$ & $\frac{\operatorname{Std}\left(X_{\mathrm{H}}\right)}{\operatorname{Std}\left(X_{\mathrm{H}}\right)}$ & $\frac{\operatorname{Std}\left(X_{\mathrm{H} 1}\right)}{\operatorname{Std}\left(X_{\mathrm{H}}\right)}$ & $\frac{\operatorname{Std}\left(X_{\mathrm{H} 2}\right)}{\operatorname{Std}\left(X_{\mathrm{H}}\right)}$ & $\frac{\operatorname{Std}\left(X_{\mathrm{H} 3}\right)}{\operatorname{Std}\left(X_{\mathrm{H}}\right)}$ & $\frac{\operatorname{Std}\left(X_{\mathrm{H} 4}\right)}{\operatorname{Std}\left(X_{\mathrm{H}}\right)}$ \\
\hline Units & $m$ & $\%$ & $\%$ & $\%$ & $\%$ & $\%$ \\
\hline Energy produced & 29.4 & 100 & 102 & 100 & 73 & 163 \\
\hline Shaft, extreme & 29.4 & 100 & 87 & 87 & 75 & 132 \\
\hline Blade F, extreme & 29.4 & 100 & 91 & 91 & 96 & 105 \\
\hline Shaft, DEL & 29.4 & 100 & 94 & 91 & 78 & 119 \\
\hline Blade F, DEL & 29.4 & 100 & 95 & 93 & 95 & 111 \\
\hline Energy produced & 72 & 100 & 97 & 87 & 563 & 157 \\
\hline Shaft, extreme & 72 & 100 & 77 & 70 & 172 & 131 \\
\hline Blade $F$, extreme & 72 & 100 & 92 & 89 & 115 & 117 \\
\hline Shaft, DEL & 72 & 100 & 92 & 85 & 216 & 119 \\
\hline Blade F, DEL & 72 & 100 & 89 & 85 & 180 & 111 \\
\hline
\end{tabular}


control system, as well as the filtering effect of the rotor where only certain frequencies in the incoming wind affect the different components. The results from the perturbation cases showed that some reduction in the uncertainty can be achieved by constraining all three turbulence components or by increasing the number of constraints in the longitudinal direction. However, considering the already low uncertainty achieved with the baseline double-rotating pattern, these improvements are only marginal. In most cases, constraining the longitudinal component of turbulence in 8-10 thousand points for a 10 min simulation should be sufficient.

The results shown in the present study represent what can be considered a best-case scenario, because they do not take into account the relation between a lidar measurement taken in real-world conditions at an arbitrary distance from the rotor and the actual $u$ component of the wind speed at the rotor plane. Thus, when applying the same method to field measurements for a load verification purpose, it can be expected that the overall load uncertainty will be higher as a result of other contributing factors such as the uncertainty in lidar measurements, but also possibly terrain properties, wind climate, load measurement system, etc.

\section{CONCLUSIONS}

In the present paper, we demonstrated a method for incorporating wind measurements from multiple-point scanning lidars into the turbulence fields serving as input to wind turbine load simulations. A numerical study showed the application of the constrained turbulence method to load simulations on a $10 \mathrm{MW}$ wind turbine model using six example lidar patterns. The results from the load calculation study showed that including lidar measurements as constraints in the turbulence field can potentially reduce the statistical uncertainty in the loads and the power production. The reduction in load uncertainty can be expected for load components which are typically affected by turbulence such as blade flapwise moments, tower fore-aft moment, yaw moment, etc. The turbulence length scale was shown to have an influence on uncertainty, with larger length scales leading to larger reductions in uncertainty as a result of increased correlation length. Constraining the longitudinal turbulence component was found to be sufficient for most purposes as the reduction in uncertainty by constraining the other two components was marginal. The overall achieved reduction in uncertainty did not fully correlate with the proportion of the variance explained by the different scanning patterns, showing that the optimal solution for a scanning pattern is governed by a combination of several factors.

\section{ACKNOWLEDGEMENT}

The work presented herein is a part of the Danish Innovation Fund (Innovationsfonden) project titled 'UniTTe-Unified Testing Procedures for Wind Turbines Through Inflow Characterization Using Nacelle Lidars', grant no. 1305-00024A. The financial support is greatly appreciated.

\section{REFERENCES}

1. Veers PS. Three-dimensional wind simulation. Sandia National Laboratories Report No. SAND88-0152, Albuquerque, New Mexico, 1988.

2. Mann J. The spatial structure of neutral atmospheric surface-layer turbulence. Fluid Mechanics 1994; 273: $141-168$.

3. Mann J. Wind field simulation. Probabilistic Engineering Mechanics 1998; 13: 269-282.

4. Bierbooms W, Cheng PW. Stochastic gust model for design calculations of wind turbines. Journal of Wind Engineering and Industrial Aerodynamics 2002; 90: 1237-1251.

5. Nielsen M, Larsen GC, Mann J, Ott S, Hansen KS, Pedersen BJ. Wind Simulation for Extreme and Fatigue Loads. Tech. Rep. Risø-R-1437(EN), Risø National Laboratory: Roskilde, Denmark, 2004.

6. Bierbooms W, Veldkamp D. Time domain comparison of simulated and measured wind turbine loads using constrained wind fields. In Proceedings of the Euromech Colloquium 'Wind Energy'. ISBN-10 3-540-33865-9 Springer, Berlin Heidelberg New York 2007.

7. Kim T, Pedersen MM, Larsen TJ. A comparison study of the two-bladed partial pitch turbine during normal operation and an extreme gust conditions. Journal of Physics: Conference Series (Online) 2014; 524: 012065.

8. Stein ML. Statistical Interpolation of Spatial Data: Some Theory for Kriging. Springer: New York, 1999.

9. Ditlevsen OD. Dimension reduction and discretization in stochastic problems by regression method. In Mathematical Models for Structural Reliability Analysis. CRC Press: Boca Raton, 1996; 51-138. 
10. Li CC, Der Kiureghian A. Optimal discretization of random fields. Journal of Engineering Mechanics 1993; 119: 1136-1154.

11. Hoffmann Y, Ribak E.Constrained realizations of Gaussian fields: a simple algorithm. The Astrophysical Journal, 380, L1-L8

12. Simley E, Pao LY. Correlation between rotating LIDAR measurements and blade effective wind speed. In Proceedings of the 51st AIAA Aerospace Sciences Meeting. Grapevine: Texas, 2013.

13. Simley E, Pao LY. LIDAR Wind speed measurements of evolving wind fields, Report No. NREL/SR-5000-55516, National Renewable Energy Laboratory, Boulder, Colorado, 2012.

14. ZephIR Lidar. ZephIR 300 and ZephIR DM brochure, 2015. http://www.zephirlidar.com/support/publications/, accessed 19-04-2016

15. Borraccino, A, Courtney, M, and Wagner, R. Generic methodology for calibrating profiling nacelle lidars. DTU Wind Energy Tech. Rep., DTU Wind Energy E; no. 0086, 2015)

16. Bak C, Zahle F, Bitsche R, Kim T, Yde A, Henriksen LC, Natarajan, A, Hansen, M. Description of the DTU 10 MW reference wind turbine. DTU Wind Energy Report-I-0092, 2013.

17. Kristensen L, Lenschow DH, Kirkegaard P, Courtney M. The spectral velocity tensor for homogeneous boundary-layer turbulence. Boundary-Layer Meteorology 1989; 47: 149-193.

18. Kragh KA, Hansen MH, Mikkelsen T. Precision and shortcomings of yaw error estimation using spinner-based light detection and ranging. Wind Energy 2013; 16: 353-366.

19. Cundy H, Rollett A. Lissajous’s figures, §5.5.3. In Mathematical Models (3rd edn). Stradbroke, England: Tarquin Pub, 1989; 242-244.

20. Larsen TJ, Hansen AM (2012) How to HAWC2, the user's manual. Tech. Rep. Risø-R-1597(ver.4-3) (EN), DTU Wind Energy, Roskilde, Denmark.

21. IEC. International Standard IEC 61400-1: Wind Turbines - Part 1: Design Guidelines, 2005, International Electrotechnical Commission, Geneva, Switzerland.

22. Chougule A, Mann J, Segalini A, Dellwik E. Spectral tensor parameters for wind turbine load modeling from forested and agricultural landscapes. Wind Energy 2013; 00: 1-12. 